\title{
O QUE COMUNICAM OS ÍNDICES DE QUALIDADE DE ÁGUA E DE ESTADO TRÓFICO EM UM RESERVATÓRIO DO SEMIÁRIDO BRASILEIRO?
}

\author{
DO WATER QUALITY AND TROPHIC STATE INDICES COMMUNICATE THE SAME STATUS \\ IN A RESERVOIR IN THE BRAZILIAN SEMIARID?
}

\author{
Gustavo Ross Ribeiro LIMA ${ }^{1}$, Stephanie de Oliveira SOUZA ${ }^{1}$, Francisco Klayton Marques \\ de ALENCAR ${ }^{1}$, Andréa Limaverde de ARAÚJO ${ }^{2}$, Fernando José Araújo da SILVA ${ }^{1}$ \\ ${ }^{1}$ Programa de Pós-graduação em Engenharia Civil do Departamento de Engenharia Hidráulica e Ambiental, POSDEHA. \\ Universidade Federal do Ceará, Centro de Tecnologia, Departamento de Engenharia Hidráulica e Ambiental. Bloco $713,1^{\circ}$ Andar, \\ Campus do Pici. Fortaleza, CE - Brasil. E-mails: gustavo_ks4@hotmail.com; stephanie_souza@ hotmail.com; \\ klayton.eq@gmail.com; fjas@deha.ufc.br \\ ${ }^{2}$ Superintendência Estadual do Meio Ambiente - SEMACE, Gerência de Monitoramento Ambiental - GEAMO. R. Jaime Benévolo, \\ 1400 - Fátima. Fortaleza - CE, Brasil. E-mail: andrea.limaverde@semace.ce.gov.br

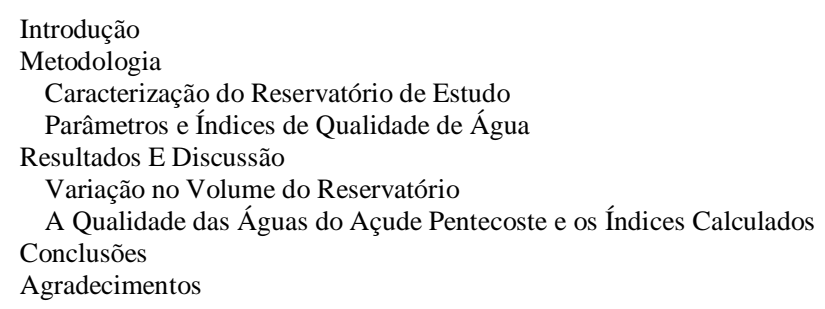

RESUMO - As águas represadas em reservatórios artificiais são vulneráveis a processos físicos, químicos e biológicos capazes de promover desequilíbrios nestes ecossistemas, como a eutrofização, em razão do elevado tempo de detenção hidráulica. Isto pode ser mais acentuado em regiões de clima semiárido. O presente trabalho buscou determinar as condições ambientais do reservatório Pereira de Miranda (açude Pentecoste), Ceará, a partir do cômputo do Índice de Qualidade de Água (IQA), Índice de Estado Trófico (IET) e Índice de Qualidade de Água de Reservatórios no Semiárido (IQARSA). A estiagem prolongada favoreceu a deterioração da qualidade da água. Isso foi apontado pela associação entre o percentual de volume disponível ou a profundidade média no reservatório com parâmetros de qualidade. Os resultados evidenciaram tendências divergentes quanto aos índices aplicados. O uso do IQA $A_{R S A}$ pode oferecer uma interpretação mais realista. Exige, porém, um maior aprimoramento, que trate de parâmetros e respectivos pesos para as ponderações.

Palavras-chave: monitoramento ambiental, deterioração qualitativa da água, eutrofização.

\begin{abstract}
Artificial reservoirs and their waters are vulnerable to physical, chemical and biological processes capable of promoting imbalances in these ecosystems, such as eutrophication, due to the high hydraulic retention time. This vulnerability is stres sed under semiarid condition. The present research focused on water quality of the Pereira de Miranda reservoir (also known as açude Pentecoste), Ceará, located in the Northeast Region of Brazil. The study used the traditional Water Quality Index (WQI), Trophic State Index (TSI) and an adapted Water Quality Index for Reservoirs in Semiarid (WQI $\mathrm{ISA}_{\mathrm{RA}}$ ). Prolonged drought favored deterioration of water quality. This was pointed out via association between available volume (as percentage of the reservoir capacity) or mean depth with water quality parameters. The results showed divergent trends regarding the applied indices. The use of WQIRSA may offer a more realistic interpretation. However, it requires improvement.
\end{abstract}

Keywords: environmental monitoring, water quality deterioration, eutrophication.

\section{INTRODUÇÃO}

A ocorrência de milhares de reservatórios no trópico semiárido brasileiro resulta de um entendimento técnico histórico. O atendimento da crescente demanda de água no semiárido brasileiro exigiu o armazenamento em reservatórios artificiais (Ammar et al., 2017). Os reservatórios do semiárido brasileiro são empregados para contornar uma situação imposta por condicionantes climáticas, como altas temperaturas e índices pluviométricos erráticos. $\mathrm{O}$ uso de corpos lênticos motiva $\mathrm{O}$ estabelecimento de formas de manejo sustentado e de gerenciamento desses ecossistemas. É importante que a qualidade das águas nos açudes seja satisfatória, a fim de que se possa atender aos requisitos qualitativos dos diferentes usos.

Os reservatórios, porém, são vulneráveis à degradação da qualidade de suas águas, em função não apenas da variabilidade climática, mas também de tensores antrópicos. Estas estruturas lênticas possuem elevado tempo de detenção hidráulica, visto que sua principal 
função é a reservação hídrica plurianual, e isso é fator que contribui ainda mais para essa vulnerabilidade. Dessa forma, o elevado tempo de detenção hidráulica em reservatórios do semiárido brasileiro intensifica processos físicos, químicos e biológicos capazes de promover desequilíbrios consideráveis nos ecossistemas lacustres (Mosley, 2015; Wiegand et al., 2016).

Há dificuldade intrínseca no controle das causas antrópicas de degradação da qualidade da água. A degradação da qualidade das águas é desafio de contornos particulares em regiões com maior pobreza hídrica. No Brasil, este contexto tem premência em seu semiárido. Para isso, torna-se necessária uma monitoração sistemática que disponibilize informações que permitam avaliar a evolução da qualidade da água no corpo hídrico e conhecer as tendências de sua variação, notadamente entre os ciclos anuais.

Com base no exposto, a utilização dos índices de qualidade de água (IQA) e de estado trófico (IET) são importantes instrumentos de avaliação da qualidade dos recursos hídricos (Andrietti et al., 2016). São utilizados para orientar classificações com base em um conjunto de parâmetros de qualidade aceitos para um fim desejado, caracterizando e simplificando o entendimento das condições do meio aquático. Servem também para identificar distúrbios e tendências de um corpo aquático em um determinado intervalo de tempo (Borges \& Guimarães, 2000; Sreedhar et al., 2017).

Devido à dinâmica trófica e à variação de parâmetros de qualidade de água e volume, próprias de corpos aquáticos de regiões de clima quente semiárido, há de se buscar abordagens próprias. Essas surgem com o propósito de melhor representar, e até mesmo esclarecer o comportamento desses ambientes aquáticos. Todavia, o desenvolvimento para tal explicação deve ponderar a complexidade de biossistemas e a condensação de informações. Ao mesmo tempo, diferentes índices podem comunicar estados diferentes quanto à satisfação de critérios de qualidade. Em tal contexto, o presente estudo surge da necessidade de se verificar a relação entre os valores de IQA e IET em águas de um reservatório no semiárido brasileiro. Além disto, o estudo contempla o efeito da estiagem sobre a qualidade da água.

\section{METODOLOGIA}

\section{Caracterização do Reservatório de Estudo}

A análise teve como objeto de estudo o reservatório Pereira de Miranda, também conhecido como açude Pentecoste, localizado entre as coordenadas (UTM) $470.964 \mathrm{mE}$ e 9.579 .848 $\mathrm{mN}$. O reservatório faz parte da bacia hidrográfica do rio Curu e foi construído entre os anos de 1950 e 1957 pelo Departamento Nacional de Obras Contra as Secas (DNOCS). O açude Pentecoste represa o rio Canindé, com bacia hidrográfica de $2.840 \mathrm{~km}^{2}$. Possui uma capacidade de armazenamento de $395.638 .000 \mathrm{~m}^{3}$, com área de bacia hidráulica próxima de 5.700 ha (Ceará, 2019).

$\mathrm{O}$ açude tem usos múltiplos, como o abastecimento de água, a irrigação, piscicultura e controle das cheias do rio Curu (DNOCS, 2019). O mapa de localização da área em estudo é ilustrado na figura 1 .

\section{Parâmetros e Índices de Qualidade de Água}

$\mathrm{O}$ monitoramento da qualidade da água para o estudo foi em ponto de coleta próximo à torre de tomada d'água (PET-01), sob as coordenadas (UTM) $471.708 \mathrm{mE}$ e $9.579 .898 \mathrm{mN}$, e elevação aproximada de $58 \mathrm{~m}$ (a.n.m.).

A coleta de amostras foi à profundidade de 0,30 $\mathrm{m}$ abaixo da superfície líquida. O período de estudo compreendeu os anos de 2004 a 2018, com amostras coletadas com frequência trimestral. Entretanto, os dados foram agrupados para representar valores semestrais.

Tal enfoque visa comparar o efeito da quadra chuvosa concentrada no primeiro semestre de cada ano, conforme perfil climático do semiárido brasileiro (Brasil, 2017).

Além dos dados de qualidade de água empregados nos índices, foram considerados também dados de precipitação (Estação ANA n ${ }^{\circ}$ 339028) durante o período de estudo. A esses dados somam-se os percentuais de volume de água disponíveis no reservatório, bem como a profundidade média do reservatório ao longo do tempo. Estes dados foram obtidos através do Portal Hidrológico do Ceará (Ceará, 2019).

A metodologia empregada para o cálculo do IQA foi adaptada do Índice de Qualidade de Água da National Sanitation Foundation (NSF) e proposta pela Companhia de Saneamento Ambiental do Estado de São Paulo (CETESB, 2017). O IQA resume as variáveis analisadas em um único número adimensional, variando de 0 a 100 . 

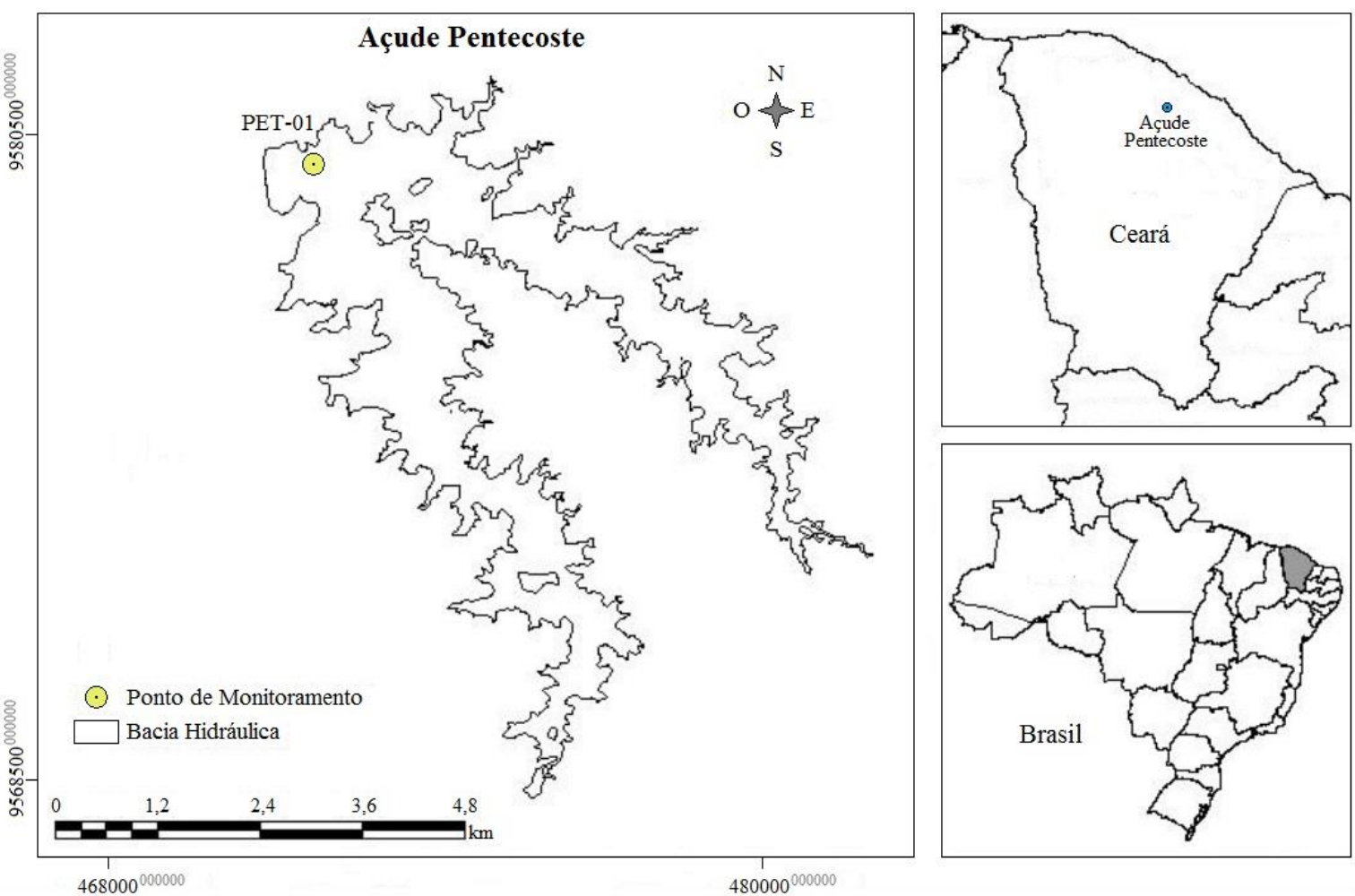

Figura 1 - Localização do açude Pentecoste, Ceará (Adaptado de França et al., 2013).

O valor do IQA foi calculado pelo produtório ponderado dos escores atribuídos a cada parâmetro de qualidade de água (Equação 1). Os parâmetros foram: temperatura da amostra $(\mathrm{T}), \mathrm{pH}$, oxigênio dissolvido (OD), demanda bioquímica de oxigênio (DBO), coliformes termotolerantes $\left(\mathrm{C}_{\mathrm{TT}}\right)$, nitrogênio total $\left(\mathrm{N}_{\mathrm{T}}\right)$, fósforo total $\left(\mathrm{P}_{\mathrm{T}}\right)$, sólidos totais (ST) e turbidez (Turb). Dessa forma, no cálculo do IQA são consideradas variáveis de qualidade que permitem, em princípio, verificar o aporte de águas poluídas no corpo aquático. Os procedimentos analíticos seguiram os métodos descritos em APHA (1998).

$$
I Q A=\prod_{i=1}^{n} q_{i}^{w_{i}}
$$

Em que: qi é a qualidade do parâmetro i obtido através da curva média especifica de qualidade e $\mathrm{w}_{\mathrm{i}}$ é o peso atribuído ao parâmetro i, em função da sua importância na qualidade, entre 0 e 1 .

Os valores de IQA são definidos em intervalos de classes, conforme a Tabela 1, e expressam a qualidade da água em determinado momento. Para o escopo do estudo aqui empreendido, a análise será com destaque maior para o ciclo anual.

O Índice de Estado Trófico (IET) avalia a qualidade da água quanto ao enriquecimento por nutrientes e seu efeito relacionado ao crescimento excessivo de algas e cianobactérias (Araújo et al.,
2013).

Tabela 1 - Classificação da qualidade de água segundo o

\begin{tabular}{|c|c|}
\hline Valor & Avaliação \\
\hline $79<\mathrm{IQA} \leq 100$ & Ótima \\
\hline $51<\mathrm{IQA} \leq 79$ & Boa \\
\hline $36<\mathrm{IQA} \leq 51$ & Razoável \\
\hline $19<\mathrm{IQA} \leq 36$ & Ruim \\
\hline IQA $\leq 19$ & Péssima \\
\hline
\end{tabular}

Para a classificação do grau de trofia, empregou-se o Índice de Estado Trófico de Cunha et al., (2013a, b). Segundo Araújo et al. (2018), o modelo desses tem maior amplitude de classes, sofre menor variação e é mais adequado aos trópicos.

Para o cálculo do IET, foram consideradas as variáveis clorofila $a(\mathrm{Cl} a)$ e fósforo total $\left(\mathrm{P}_{\mathrm{T}}\right)$. O modelo não emprega valores de transparência (i.e. profundidade de disco de Secchi), pois este parâmetro pode ser afetado pela elevada turbidez decorrente do material mineral em suspensão, e não apenas pela densidade de organismos planctônicos, não representando o real estado de trofia existente (Buiteveld, 1995; Effler et al., 2017).

O cômputo do IET total foi feito a partir da média aritmética do IET parcial para a clorofila $a$ $\left(\right.$ IET $\left._{\mathrm{Cl} a}\right)$ e do IET parcial para o fósforo total

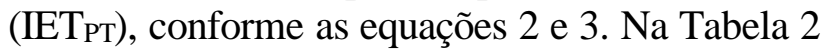
são apresentados os valores e as classes referentes 
ao IET.

$$
\begin{aligned}
& I E T_{C i a}=3,624 \cdot \ln (\mathrm{Cla})+47,850 \\
& I E T_{P_{\tau}}=3,987 \cdot \ln \left(P_{T}\right)+40,815
\end{aligned}
$$

Em que: Cla é a concentração de clorofila $a \mathrm{em}$ $\mu \mathrm{g} / \mathrm{L}$ e $\mathrm{P}_{\mathrm{T}}$ é a concentração de fósforo total em $\mu \mathrm{g}$ $\mathrm{P} / \mathrm{L}$. Ao final, os resultados obtidos foram classificados de acordo com a Tabela 2.

Tabela 2 - Valores e classes do Índice de Estado Trófico (IET).

\begin{tabular}{c|c|c|c}
\hline Classificação & Valor do IET & $\begin{array}{c}\text { Fósforo total } \\
(\boldsymbol{\mu g} / \mathbf{L})\end{array}$ & $\begin{array}{c}\text { Clorofila a } \\
(\boldsymbol{\mu g} / \mathbf{L})\end{array}$ \\
\hline Ultraoligotrófico & IET $\leq 51,1$ & $\mathrm{P}_{\mathrm{T}} \leq 15,9$ & $\mathrm{Cl} a \leq 2,0$ \\
\hline Oligotrófico & $51,2 \leq \mathrm{IET} \leq 53,1$ & $16,0 \leq \mathrm{P}_{\mathrm{T}} \leq 23,8$ & $2,1 \leq \mathrm{Cl} a \leq 3,9$ \\
\hline Mesotrófico & $53,2 \leq \mathrm{IET} \leq 55,7$ & $23,9 \leq \mathrm{P}_{\mathrm{T}} \leq 36,7$ & $4,0 \leq \mathrm{Cl} a \leq 10,0$ \\
\hline Eutrófico & $55,8 \leq \mathrm{IET} \leq 58,1$ & $36,8 \leq \mathrm{P}_{\mathrm{T}} \leq 63,7$ & $10,1 \leq \mathrm{Cl} a \leq 20,2$ \\
\hline Supereutrófico & $58,2 \leq \mathrm{IET} \leq 59,0$ & $63,7 \leq \mathrm{P}_{\mathrm{T}} \leq 77,6$ & $20,3 \leq \mathrm{Cl} a \leq 27,1$ \\
\hline Hipereutrófico & IET $\geq 59,1$ & $\geq 77,7$ & $\geq 27,2$ \\
\hline \multicolumn{4}{c}{ Fonte: Cunha et al. $(2013 \mathrm{a}, \mathrm{b})}$.
\end{tabular}

Além do IQA e do IET, calculou-se também o Índice de Qualidade de Água em Reservatórios $\left(\mathrm{IQA}_{\mathrm{R}}\right)$. Esse índice é, desde 2005, empregado no estado do Paraná, região sul do país. Tenta contemplar tanto o caráter limnológico do reservatório quanto a qualidade das águas e sua tendência ao longo do tempo. Assim, o IQAR auxilia na avaliação e classificação das águas dos reservatórios conforme o grau de degradação (IAP, 2009). No âmbito do presente estudo, empregou-se o IQARSA (Índice de Qualidade de Água de Reservatórios no Semiárido) como adaptação do índice originalmente empregado pelo Instituto Ambiental do Paraná (IAP).

A Tabela 3 contém os seguintes parâmetros adicionais ao IQA e ao IET empregados para o cálculo do IQARSA: módulo da variação da concentração de oxigênio dissolvido em torno do valor de saturação $\left(\Delta \mathrm{OD}_{\mathrm{SAT}}\right)$, nitrogênio inorgânico $\left(\mathrm{N}_{\mathrm{ING}}=\right.$ amônia total $(\mathrm{AMT})+$ nitrato $\left(\mathrm{NO}_{3}^{-}\right)+$nitrito $\left(\mathrm{NO}_{2}^{-}\right)$), percentual do volume de água disponível no reservatório (VD), profundidade média no reservatório $\left(\mathrm{H}_{\mathrm{M}}\right)$ e logaritmo da contagem de células de cianobactérias $\left(\log _{10}\right.$ CIAN). A tabela apresenta ainda os escores associados às classes de grau de degradação refletida pelos valores observados através de cada parâmetro, bem como o peso destes. $\mathrm{O}$ cômputo do índice é procedido através de média ponderada dos escores das classes (Equação 4). Por fim, a Tabela 4 traz a classificação do grau de degradação com base no IQARSA.

$$
I Q A_{R S A}=\sum_{i=1}^{n} q_{i} \cdot w_{i}
$$

\begin{tabular}{|c|c|c|c|c|c|c|}
\hline \multirow{2}{*}{ Variáveis } & \multirow{2}{*}{ Unidade } & \multirow{2}{*}{ Peso } & \multicolumn{4}{|c|}{ Classes de intervalos } \\
\hline & & & 1 & 2 & 3 & 4 \\
\hline$\Delta \mathrm{OD}_{\mathrm{SAT}}$ & $\%$ & 0,19 & $\leq 15$ & $16-25$ & $26-40$ & $>40$ \\
\hline $\mathrm{P}_{\mathrm{T}}$ & $\mu \mathrm{g} / \mathrm{L} \mathrm{P}$ & 0,14 & $\leq 10$ & $11-30$ & $31-50$ & $>50$ \\
\hline $\mathrm{N}_{\mathrm{ING}}$ & $\mathrm{mg} / \mathrm{L} \mathrm{N}$ & 0,09 & $\leq 0,50$ & $0,51-1,00$ & $1,01-2,00$ & $>2,00$ \\
\hline $\mathrm{Cl} a$ & $\mu \mathrm{g} / \mathrm{L}$ & 0,17 & $\leq 5$ & $6-15$ & $16-25$ & $>25$ \\
\hline DBO & $\mathrm{mg} / \mathrm{L}$ & 0,14 & $\leq 3$ & $3-5$ & $6-10$ & $>10$ \\
\hline $\mathrm{VD}$ & $\%$ & 0,11 & $>80$ & $80-60$ & $59-20$ & $<20$ \\
\hline $\mathrm{H}_{\mathrm{M}}$ & $\mathrm{m}$ & 0,07 & $>20$ & $20-10$ & $9-3$ & $<3$ \\
\hline Cianobactérias & $\log _{10}($ células/mL $)$ & 0,09 & $<4,0$ & $4,1-4,4$ & $4,5-4,8$ & $>4,8$ \\
\hline
\end{tabular}

Tabela 3 - Parâmetros, pesos, classes e intervalos referentes ao Índice de Qualidade de Água de Reservatórios no Semiárido (IQA $\left.A_{R S A}\right)$.

Tabela 4 - Valores e estados de classificação do Índice de Qualidade de Água de Reservatórios (IQARSA).

\begin{tabular}{c|c|c}
\hline Valor do IQARSA & Classe & Grau de degradação \\
\hline $1,0-1,4$ & 1 & Não degradado a pouco degradado \\
\hline $1,5-2,4$ & 2 & Pouco a moderadamente degradado \\
\hline $2,5-3,4$ & 3 & Moderadamente a degradado \\
\hline $3,5-4,0$ & 4 & Degradado a muito degradado \\
\hline \multicolumn{2}{r|}{ Fonte: adaptado de IAP (2017). }
\end{tabular}




\section{RESULTADOS E DISCUSSÃO}

\section{Variação no Volume do Reservatório}

$\mathrm{O}$ volume hídrico disponível (VD) no açude Pentecoste teve redução acentuada a partir de 2012. É possível descrever tal processo em dois períodos. No primeiro período, de 2004 a 2011, o volume médio disponível foi de $63,7 \%$ da capacidade do reservatório. O percentual médio de redução desse volume foi de $0,4 \%$ ao ano, com elevações em 2006, 2008, 2009 e 2011. Esses acréscimos resultaram de quadras chuvosas acima da média, especialmente em 2009, cujo excedente foi em torno de $88,8 \%$ acima da média (normal $=839 \mathrm{~mm} / \mathrm{ano}$ ). No intervalo posterior de 2012 a 2018 - o volume médio no reservatório foi de $8,8 \%$, com percentual de redução de aproximadamente $8,5 \%$ ao ano. Nesse segundo intervalo, a redução média de precipitação em relação à normal foi por volta de $33,8 \%$. A quadra resultou essencialmente de uma estiagem prolongada de seis anos (2012 a 2017), com precipitações anuais abaixo da média. Apenas o ano de 2018 foi ligeiramente superior $(915 \mathrm{~mm})$ à normal. Os anos mais críticos foram 2012 (247 $\mathrm{mm})$ e 2016 (475 mm).

A Figura 2 mostra a variação do volume anual disponível no açude Pentecoste e a precipitação ocorrida no período. Aqui a representação em ciclo anual é bem justificada por não ter sido observada diferença significativa entre volumes médios registrados nos primeiros semestres de cada ano em relação aos do segundo semestre. Empregou-se na comparação, tanto o teste ANOVA quanto o de Kruskal-Wallis para um nível de significância de 0,05.

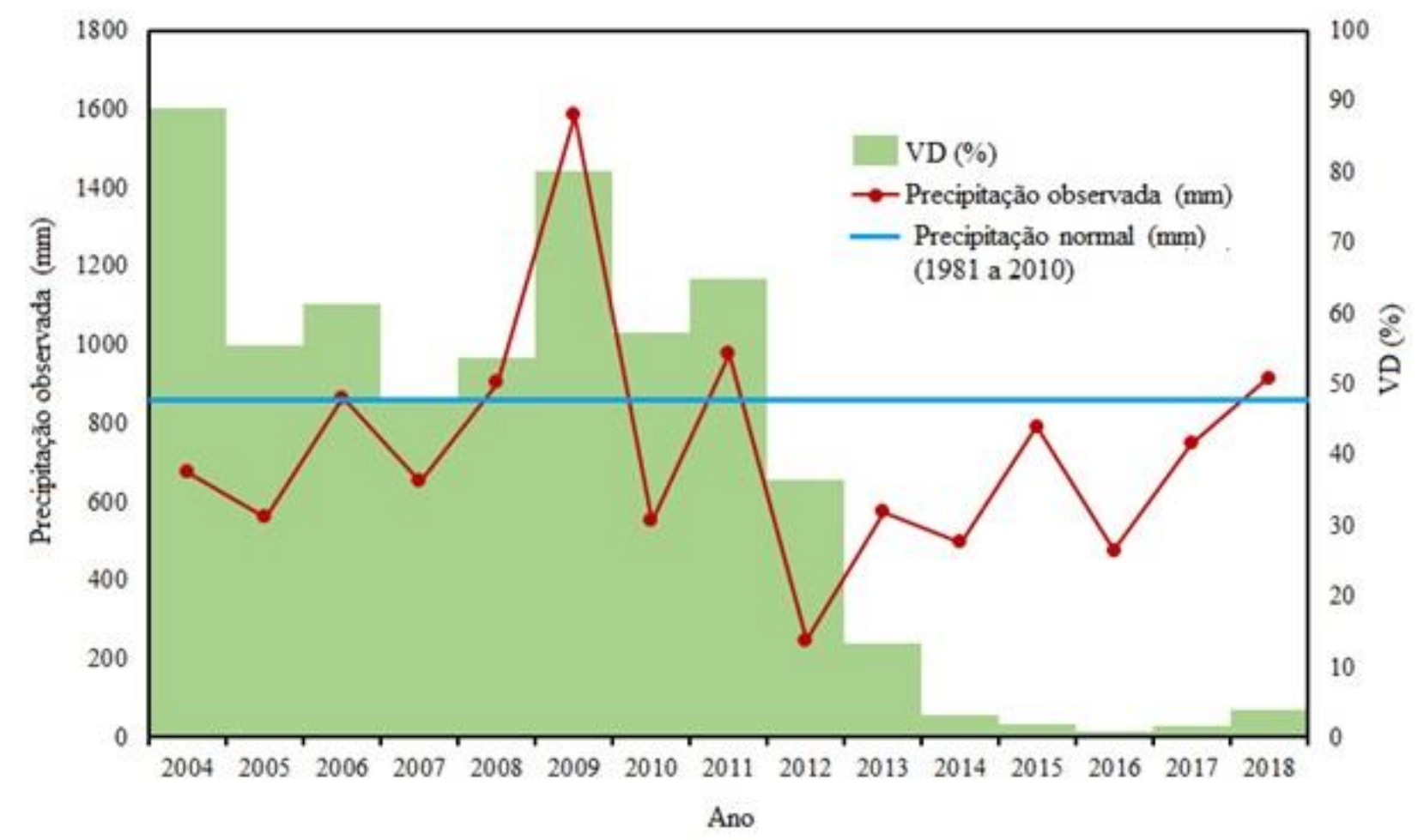

Figura 2. Variação média anual do percentual de volume hídrico e da precipitação observada no período de estudo.

\section{A Qualidade das Águas do Açude Pentecoste e os Índices Calculados}

A Tabela 5 contém um sumário descritivo dos parâmetros empregados no estudo. $\mathrm{Na}$ comparação entre os valores semestrais, não houve diferença significativa para nenhum dos parâmetros. A comparação foi conduzida tanto com o teste ANOVA quanto o de KruskalWallis para um nível de significância de 0,05.

Dentre os critérios constantes na resolução CONAMA 357/05, foram verificados valores em desacordo com a norma no caso dos parâmetros: $\mathrm{P}_{\mathrm{T}}, \mathrm{CIAN}, \mathrm{DBO}, \mathrm{Cl} a$, OD e AMT. Os graus de não conformidade foram de 96,7; 73,$3 ; 40,0 ; 30,0 ; 16,7$ e $3,3 \%$, respectivamente, considerados com base nas médias semestrais.

Os valores médios semestrais do IQA, do

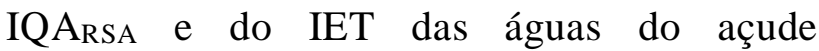
Pentecoste estão na Tabela 6. Não houve diferença estatística significativa (com $\alpha=$ 0,05 ) entre os valores observados em cada primeiro semestre, comparado aos de segundo semestre. Os resultados mostraram que, enquanto a qualidade da água predomina como 
Boa com base no IQA, pelo IET preponderam as classificações Supereutrófico e Hipereutrófico. Apesar dos conceitos de IQA e IET serem distintos quanto aos seus propósitos, os índices convergem à ideia de qualidade da água no corpo aquático. Comunicam, porém, estados diferentes.

Tabela 5 - Estatística descritiva dos parâmetros de qualidade de água, volume operacional e profundidade média no açude Pentecoste (2004 a 2018).

\begin{tabular}{c|c|c|c|c|c}
\hline Parâmetro & Unidade & Média & Mediana & $\sigma$ & Intervalo \\
\hline Temperatura & ${ }^{\circ} \mathrm{C}$ & 28,8 & 28,6 & 0,5 & $27,8-30,0$ \\
\hline $\mathrm{pH}$ & - & 8,23 & 8,29 & 0,38 & $7,58-9,01$ \\
\hline $\mathrm{C}_{\mathrm{TT}}$ & $\mathrm{NMP} / 100 \mathrm{~mL}$ & $4,2 \mathrm{E}+01$ & $8,4 \mathrm{E}+00$ & $1,0 \mathrm{E}+02$ & $1,0 \mathrm{E}+00-5,0 \mathrm{E}+02$ \\
\hline $\mathrm{DBO}$ & $\mathrm{mg} / \mathrm{L}$ & 5 & 5 & 2 & $3-11$ \\
\hline $\mathrm{OD}$ & $\mathrm{mg} / \mathrm{L}$ & 6,7 & 7,0 & 1,8 & $2,6-10,4$ \\
\hline $\mathrm{N}_{\mathrm{T}}$ & $\mathrm{mg} / \mathrm{L} \mathrm{N}$ & 2,35 & 1,68 & 1,67 & $0,76-7,85$ \\
\hline $\mathrm{P}_{\mathrm{T}}$ & $\mathrm{mg} / \mathrm{L} \mathrm{P}$ & 0,19 & 0,15 & 0,11 & $0,02-0,47$ \\
\hline $\mathrm{Turb}$ & $\mathrm{uT}$ & 18,2 & 11,7 & 17,8 & $2,8-71,9$ \\
\hline $\mathrm{ST}$ & $\mathrm{mg} / \mathrm{L}$ & 532 & 337 & 421 & $217-2025$ \\
\hline $\mathrm{Cl}$ & $\mu \mathrm{g} / \mathrm{L}$ & 34,3 & 16,5 & 36,7 & $5,7-130,4$ \\
\hline $\mathrm{AMT}$ & $\mathrm{mg} / \mathrm{L} \mathrm{N}$ & 0,26 & 0,23 & 0,13 & $0,10-0,61$ \\
\hline $\mathrm{NO}_{3}{ }^{-}$ & $\mathrm{mg} / \mathrm{L} \mathrm{N}$ & 0,42 & 0,37 & 0,20 & $0,12-0,98$ \\
\hline $\mathrm{NO}_{2}{ }^{-}$ & $\mathrm{mg} / \mathrm{L} \mathrm{N}$ & 0,049 & 0,013 & 0,061 & $0,001-0,264$ \\
\hline $\mathrm{N}_{\mathrm{ING}}$ & $\mathrm{mg} / \mathrm{L} \mathrm{N}$ & 0,74 & 0,74 & 0,25 & $0,30-1,48$ \\
\hline $\mathrm{Cianobactérias}$ & células/mL & $1,5 \mathrm{E}+05$ & $1,3 \mathrm{E}+05$ & $1,2 \mathrm{E}+05$ & $1,5 \mathrm{E}+04-5,0 \mathrm{E}+05$ \\
\hline $\mathrm{VD}$ & $\%$ & 38,1 & 45,1 & 31,2 & $0,9-94,9$ \\
\hline $\mathrm{H}_{\mathrm{M}}$ & $\mathrm{m}$ & 9,6 & 12,0 & 5,2 & $2,1-16,3$ \\
\hline
\end{tabular}

Tabela 6 - Resultados médios de IQA, IQA ${ }_{R S A}$ e IET no açude Pentecoste.

\begin{tabular}{|c|c|c|c|c|c|c|}
\hline \multirow{2}{*}{ Ano } & \multicolumn{3}{|c|}{ Índices no Primeiro Semestre } & \multicolumn{3}{|c|}{ Índices no Segundo Semestre } \\
\hline & $I Q A$ & $I Q A_{R S A}$ & IET & $I Q A$ & $I Q A_{R S A}$ & IET \\
\hline 2004 & 69 & 2,2 & 60,5 & 74 & 2,2 & 59,6 \\
\hline 2005 & 73 & 2,6 & 59,1 & 73 & 2,4 & 59,6 \\
\hline 2006 & 76 & 2,3 & 58,4 & 76 & 2,5 & 57,6 \\
\hline 2007 & 75 & 2,6 & 59,1 & 72 & 2,4 & 59,9 \\
\hline 2008 & 73 & 2,4 & 56,5 & 70 & 2,6 & 58,3 \\
\hline 2009 & 73 & 2,2 & 58,6 & 79 & 2,4 & 58,8 \\
\hline 2010 & 71 & 2,4 & 58,5 & 79 & 2,2 & 54,6 \\
\hline 2011 & 73 & 2,4 & 58,7 & 73 & 2,3 & 58,7 \\
\hline 2012 & 75 & 2,5 & 60,9 & 84 & 2,7 & 59,8 \\
\hline 2013 & 76 & 3,2 & 61,0 & 77 & 3,0 & 61,7 \\
\hline 2014 & 60 & 3,4 & 63,2 & 50 & 3,1 & 62,9 \\
\hline 2015 & 69 & 3,4 & 63,1 & 71 & 3,0 & 60,7 \\
\hline 2016 & 65 & 3,3 & 62,0 & 58 & 3,3 & 64,2 \\
\hline 2017 & 48 & 3,5 & 64,8 & 69 & 2,9 & 58,8 \\
\hline 2018 & 58 & 3,0 & 59,0 & 66 & 3,0 & 61,6 \\
\hline
\end{tabular}

Os resultados de IQA indicam que as águas do açude Pentecoste seriam adequadas a usos múltiplos e equivalentes ou bem próximos aos descritos para águas classe 2 e 3 , de acordo com a CONAMA 357/05. Já o IET aponta limitações no uso da água do reservatório, 
notadamente para abastecimento humano, mesmo com tratamento convencional. Ressalta-se que a produtividade primária elevada está associada à presença de cianobactérias e, portanto, potencialmente à ocorrência de cianotoxinas (Chislock et al., 2014; Sulis et al., 2014; Marreto et al., 2017).

A interpretação dos resultados dos IQA RSA ficou situada entre a do IQA e a do IET. Os resultados do IQARSA apontaram status de "moderadamente a degradado" das águas, porém alternando com o estado de "pouco a moderadamente degradado" em 40\% das observações.

Para analisar o grau de associação entre duas variáveis, utilizou-se o coeficiente de correlação de Pearson ( $r$ ) com nível de significância de 0,05 (Asuero et al., 2006). As correlações entre o IQA e os demais índices foram $-0,642$ com o IQA RSA $_{\text {e }}-0,656$ com o IET. O sinal negativo explica o fato de o IQA representar melhor qualidade com a elevação de seu valor, enquanto que no IET e IQARSA valores mais elevados representam pior qualidade. Para esses valores de $r$, há que se considerar não apenas o fato de que os índices têm conceitos distintos, mas também diferentes conjuntos de parâmetros agregados que os compõem. O coeficiente de correlação entre IET e o IQARSA foi um pouco mais elevado ( $r$ $=0,782)$, justificado pela interface dos parâmetros componentes $\left(\mathrm{P}_{\mathrm{T}}\right.$ e $\left.\mathrm{Cl} a\right)$.

Quanto aos parâmetros de qualidade da água, destaca-se a turbidez, que representa a atenuação do feixe de luz ao atravessar a massa líquida. No presente estudo, o parâmetro Turb correlacionou-se positivamente com os parâmetros $\mathrm{Cl} a, \mathrm{ST}$ e $\mathrm{N}_{\mathrm{T}}$ (valores de $r$ iguais a 0,$905 ; 0,800$ e 0,685 , respectivamente). A evidência de que a biomassa algal foi a principal causa de turbidez nas águas do açude Pentecoste é ratificada em correlação cruzada com ST $(r=0,841)$ e $\mathrm{N}_{\mathrm{T}}(r=0,776)$. Nesse último parâmetro, se descontada a parcela inorgânica, tem-se o nitrogênio orgânico $\left(\mathrm{N}_{\mathrm{ORG}}\right)$ que apresentou boa associação com $\mathrm{Cl} a$ $(r=0,789)$.

Em paralelo, a turbidez provocada pela lixiviação de material orgânico e inorgânico quando associada à ação dos ventos pode provocar a ressuspensão de sedimento, que contribui para recolocar em circulação os nutrientes, principalmente o fósforo, componente do cálculo do IET (Cunha et al., 2013a, b).

A presença de sólidos totais em excesso pode tanto alterar a incidência luminosa na água interferindo na realização da fotossíntese pelos organismos autotróficos, reduzindo a produção de oxigênio, como o conteúdo salino presente pode reduzir a solubilidade do oxigênio dissolvido na água. $\mathrm{O}$ oxigênio dissolvido é um importante indicador de qualidade da água, já que baixas concentrações podem indicar processos de eutrofização e prejuízo dos processos bioquímicos e biológicos, como oxidação da matéria orgânica, respiração e processos metabólicos celulares (Noriega et al., 2005).

É importante destacar que a variabilidade da precipitação na região tem acentuado efeito sobre o tempo de residência das águas acumuladas, bem como sobre o valor de VD. A redução do volume implica redução da profundidade média da coluna liquida no reservatório. Ainda assim, não há correlação destacável entre a dimensão da precipitação anual e o volume de água disponível no açude. Perdas por evaporação, evapotranspiração, infiltração e passos de tempo distintos entre o volume precipitado e o processo de acumulação impedem verificar tal correlação. Não se observou, portanto, efeito da precipitação sobre os parâmetros de qualidade.

Um reducionismo físico pode ser atribuído às variáveis $\mathrm{VD}$ e $\mathrm{H}_{\mathrm{M}}$, com efeito, principalmente, sobre os parâmetros Turb, OD, ST, $\mathrm{N}_{\mathrm{T}}$ e Cla. Isto é mostrado na Figura 3, em que se verifica que o efeito da profundidade média $\left(\mathrm{H}_{\mathrm{M}}\right)$ é mais significativo. Mutatis mutandis, a Figura 4 mostra a correlação de $\mathrm{H}_{\mathrm{M}}$ com os índices de qualidade de água estudados. A autocorrelação de $\mathrm{H}_{\mathrm{M}}$ com o IQARSA evidencia um maior valor do coeficiente de correlação.

O que ficou claro é que a estiagem prolongada favoreceu a deterioração da qualidade da água como um todo, concordando com ArenasSánchez et al. (2016). A baixa associação de significados entre IET e IQA resulta do baixo peso de $\mathrm{N}_{\mathrm{T}}$ e $\mathrm{P}_{\mathrm{T}}$ no cômputo desse do segundo índice. Quanto a isso, ressalta-se o entendimento de Piasentin et al. (2009), em que o IQA não é um indicador do nível de degradação do corpo hídrico, enquanto o IET reflete uma elevada produtividade primária do corpo hídrico. Foi isso que ocorreu no açude Pentecoste. O IQARsA, por seu turno, apareceu como índice intermediário 
com potencial de aplicação, merecendo maior atenção.

\section{CONCLUSÕES}

Os resultados do estudo mostraram domínio da classe Boa, com IQA médio igual a 70. O IET (valor médio $=60$ ) indicou variações entre os estados Supereutrófico e Hipereutrófico, com risco para a qualidade da água a ser consumida, mesmo após tratamento convencional. A interpretação dos resultados dos IQARSA (valor médio $=2,7$ ) ficou situada entre a do IQA e a do IET. Os resultados do IQARSA apontaram status de "moderadamente a degradado" das águas, porém alternando com o estado de "pouco a moderadamente degradado".

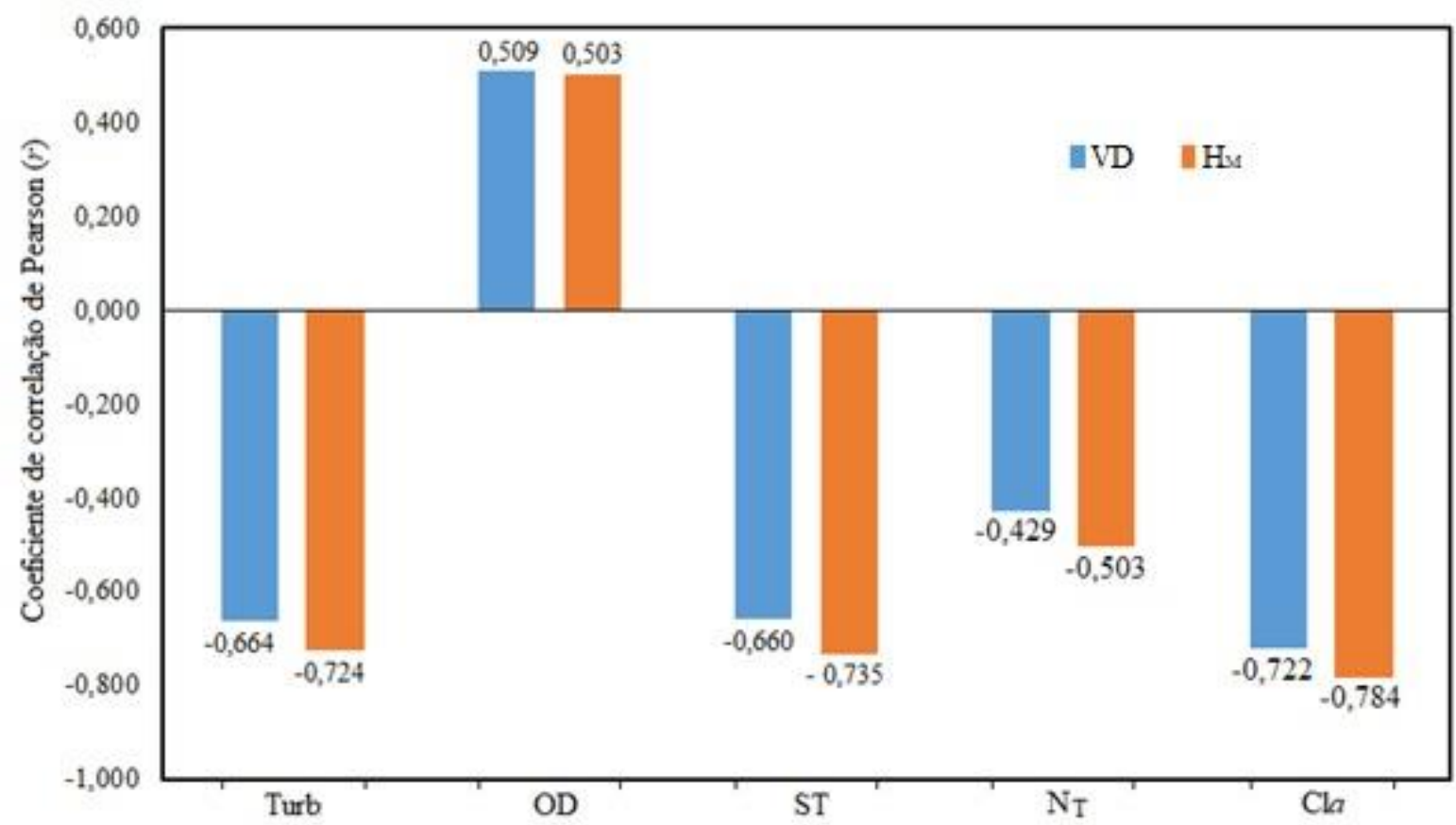

Figura 3 - Valores dos coeficientes de correlação de Turb, OD, ST, N $\mathrm{N}_{\mathrm{T}}$ e Cla com as variáveis de estado operacional VD e $\mathrm{H}_{\mathrm{M}}$ no açude Pentecoste.

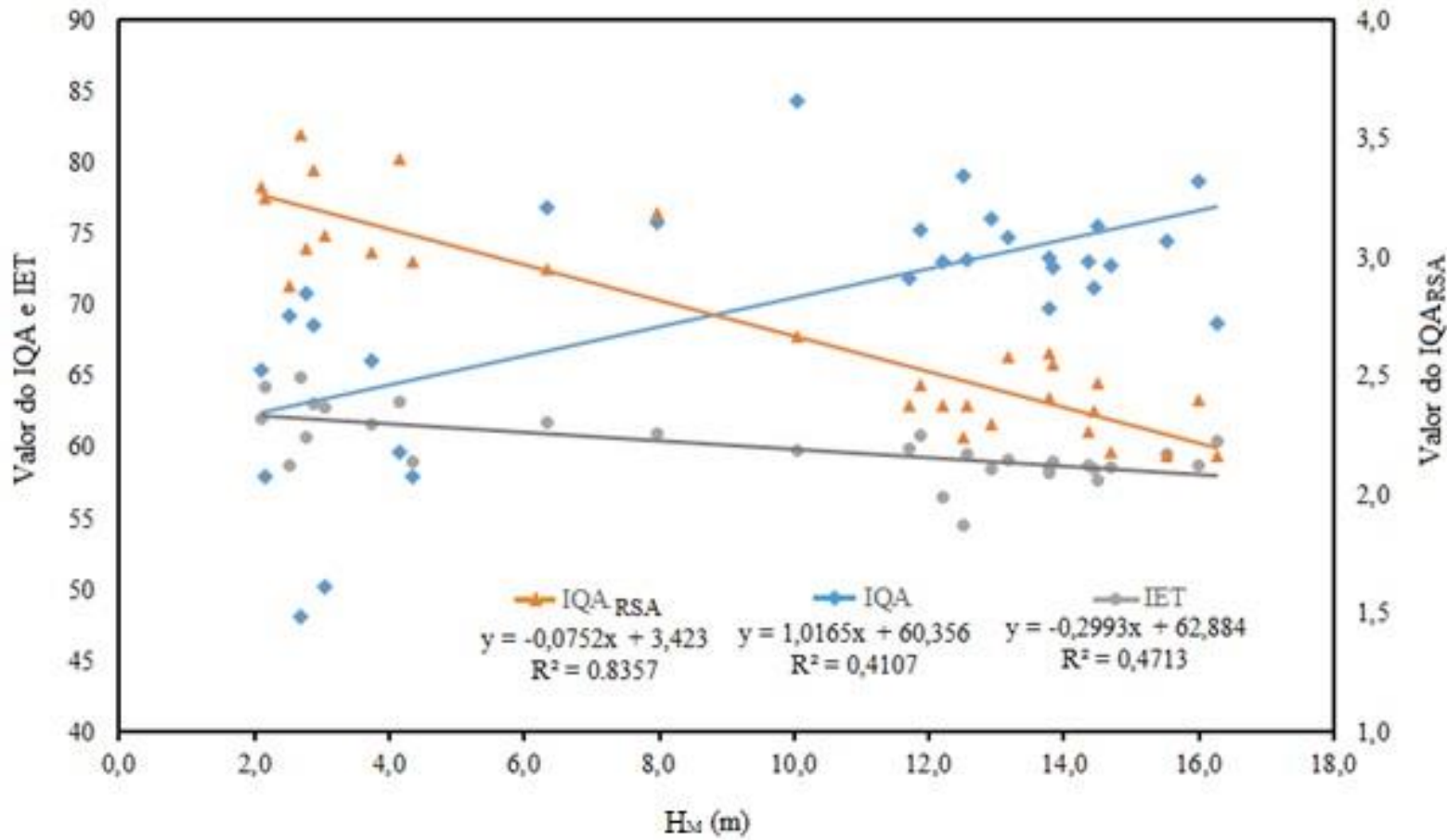

Figura 4 - Correlação entre a profundidade média no açude Pentecoste e os índices IQA, IET e IQARSA.

O estudo mostrou ainda que não houve correlação significativa entre o IQA e o IET nas águas do açude Pentecoste no intervalo de tempo considerado.

Ademais, a avaliação isolada do IQA não é indicada para determinar o estado de 
conservação do reservatório em questão, sob o risco de oscilações dos parâmetros do referido índice serem atenuadas umas pelas outras em razão das sobreposições. O uso do IQARSA pode oferecer uma interpretação mais realista. Exige, porém, um maior aprimoramento, que trate de parâmetros e respectivos pesos para as ponderações.
Nos ciclos pluviométricos anuais, a distinção semestral representada pelos períodos chuvoso e seco não apontou influência sobre os valores dos parâmetros de qualidade de água, bem como os índices. No entanto, verificou-se deterioração da qualidade da água com a redução do volume disponível no reservatório, bem como da profundidade média.

\section{AGRADECIMENTOS}

À Companhia de Gestão dos Recursos Hídricos (COGERH) pela disponibilização dos dados de qualidade de água utilizados neste trabalho. À Coordenação de Aperfeiçoamento de Pessoal de Nível Superior (CAPES) e à Fundação Cearense de Apoio ao Desenvolvimento Científico e Tecnológico (FUNCAP) pelas bolsas de mestrado concedidas aos autores.

\section{REFERÊNCIAS}

AMMAR, R.; KAZPARD, V.; EL SAMRANI, A. G.; AMACHA, N.; SAAD, Z.; CHOU, L. Hydrodynamic influence on reservoir sustainability in semi-arid climate: A physicochemical and environmental isotopic study. Journal of Environmental Management, v. 197, p. 571-581, 2017.

ANDRIETTI, G.; FREIRE, R.; AMARAL, A.G.; ALMEIDA, F.T.; BONGIOVANI, M.C.; SCHNEIDER, R.M. Índices de qualidade da água e de estado trófico do rio Caiabi, MT. Rev. Ambient. Água, v. 11, n. 1, p. 162-175, 2016.

APHA. Standard Methods for the examination of water and wastewater. 20th ed., American Public Health Association, American Water Works Association, Water Environmental Federation, Washington, 1998. 937pp.

ARAÚJO, D.R.; MENDONÇA, A.S.F.; REIS, J.A.T. Análise de variação e comparação de índices de estado trófico: reservatórios dos aproveitamentos hidrelétricos de Rio Bonito e Suíça. Eng. Sanit. Ambient. v. 23 n. 1, p. 55-52, Jan./Fev., 2018.

ARAÚJO, J.A.F.; SALES, R.J.M.; SOUZA, R.O. Risco de eutrofização em reservatórios de regiões semiáridas com uso da teoria dos conjuntos difusos. REGA, v. 10, n. 1, p. 29-39, 2013. ARENAS-SÁNCHEZ, A.; RICO, A.; VIGHI, M. Effects of water scarcity and chemical pollution in aquatic ecosystems: state of art. Science of the Total Environment, v. 572, p. 390-403, December, 2016.

ASUERO, A.G., SAYAGO, A.; GONZÁLEZ, A.G. The Correlation Coefficient: An Overview. Critical Reviews in Analytical Chemistry, 36(1), 41-59, 2006.

BORGES, J.T. \& GUIMARÃES, J.R. Utilização do índice de qualidade de águas (IQA - CETESB) e do índice de estado trófico (IET - CARLSON) para classificar a qualidade das águas da lagoa do Taquaral - Campinas-SP. In: REUNIÃO ANUAL DA SOCIEDADE BRASILEIRA DE QUÍMICA, 23. 2000. Poços de Caldas. Anais eletrônicos. Disp. em: <http://www.sbq.org.br/ranteriores/23/resumos/03861/index.html>. Acesso em: 28 mai. 2019.

BRASIL. Agência Nacional de Águas (ANA). Reservatórios do Semiárido Brasileiro: Hidrologia, Balanço Hídrico e Operação. Relatório Síntese. Agência Nacional de Águas (ANA), Brasília, 2017. 88 p.

BRASIL. Ministério do Meio Ambiente, Conselho Nacional do Meio Ambiente (CONAMA). Resolução CONAMA no 357, de 17 de março de 2005. Disp. em: <http://www.mma.gov.br>. Acesso em: 07 mai. 2019.

BUITEVELD, H. A model for calculation of diffuse light attenuation (PAR) and Secchi depth. Netherlands Journal of Aquatic Ecology, v. 29, n. 1, p. 55-65, 1995.

CEARÁ. Companhia de Gestão dos Recursos Hídricos do Estado do Ceará (COGERH). Atlas dos Recursos Hídricos do Ceará Disp. em: <http://atlas.cogerh.com.br>. Acesso em: 20 jun.
2019.

CEARÁ. Fundação Cearense de Meteorologia e Recursos Hídricos (FUNCEME). Portal Hidrológico do Ceará. Disp. em: < http://www.hidro.ce.gov.br>. Acesso em: 11 jun. 2019.

CHISLOCK, M.F.; SHARP, K.L.; WILSON, A.E. Cylindrospermopsis raciborskii dominates under very low and high nitrogen-to-phosphorus ratios. Water Research, v., 49, n. 1, p. 207-214, February, 2014.

COMPANHIA AMBIENTAL DO ESTADO DE SÃO PAULO. Apêndice D - Índices de Qualidade das Águas. São Paulo: CETESB, 2017. Disp. em: <http://cetesb.sp.gov.br/aguasinteriores/wpcontent/uploads/sites/12/2013/11/Ap\%C3\%AAndice-D\%C3\%8Dndices-de-Qualidad e-das-\%C3\%81guas-1.pdf $>$. Acesso: em: 21 mai. 2019

CUNHA, D.G.F.; CALIJURI, M.C.; LAMPARELLI, M.C. A trophic state index for tropical/subtropical reservoirs (TSItsr). Ecological Engineering, v. 60, n. 1, p. 126-134, 2013 a.

CUNHA, R. W.; GARCIA Jr, M. D.; ALBERTONI, E. F.; PALMA-SILVA, C. Qualidade de água de uma lagoa rasa em meio rural no sul do Brasil. Revista Brasileira de Engenharia Agricola e Ambiental - Agriambi, v. 17, n. 7, 2013b.

DEPARTAMENTO NACIONAL DE OBRAS CONTRA A SECA. Arquivo Técnico. Processos do açude Pereira de Miranda. Disp. https://www.dnocs.gov.br/ dnocs/doc/canais/barragens/Barrag em\%20do\%20Ceara/pentecoste.htm >. Acesso em: 20 mai. 2019.

EFFLER, S. W. et al. A Mechanistic Model for Secchi Disk Depth, Driven by Light Scattering Constituents. Water Air Soil Pollut, v. 228, p. 153, 2017.

FRANÇA, J.M.B.; WACHHOLZ, F.; CARNEIRO NETO, J.A.; PAULINO, W.D. Comportamento das variáveis qualitativas do açude Pereira de Miranda-Pentecoste/CE, no período de estiagem. Geociências, UNESP, v. 32, n. 4, p. 586-599, 2013.

IAP. Monitoramento da qualidade das águas dos reservatórios do Estado do Paraná no período de 2005 a 2009. Relatório Técnico. Governo do Estado do Paraná. Secretaria de Estado do Meio Ambiente e Recursos Hídricos. IAP - Instituto Ambiental do Paraná. Curitiba-PR. 2009. 120p + ANEXOS

IAP. Qualidade das águas dos reservatórios do Estado do Paraná. Relatório Técnico. Governo do Estado do Paraná. Secretaria de Estado do Meio Ambiente e Recursos Hídricos. IAP - Instituto Ambiental do Paraná. Curitiba-PR. 2017. 150p + ANEXOS.

MARRETO, R.N.; BAUMGARTEN, M.G.Z; WALLNERKERSANACH, M. Trophic quality of waters in the Patos Lagoon estuary: a comparison between its margins and the port channel located in Rio Grande, RS, Brazil. Limnologica Acta 
Brasiliensia, v. 29, p. 11-12, 2017.

MOSLEY, L.M. Drought impacts on the water quality of freshwater systems; review and integration. Earth-Science Reviews, v. 140, p. 203-214, 2015.

NORIEGA, C.D.; COSTA, K.M.P.; FEITOSA, F.A.N.; MONTES, M.J.F.; GREGO, C.K.S.; SOARES, G.S.S; SILVA,

H.P. Distribuição espacial da biomassa fitoplanctônica e sua relação com os sais nutrientes, no sistema estuarino de Barra das jangadas (Pernambuco-Brasil). Arquivos de Ciências do Mar, v. 38, n. 1-2, p. 5-18, 2005.

PIASENTIN, A.M.; SEMENSATTO Jr., D.L.; SAAD, A.R.; MONTEIRO JUNIOR, A. J.; RACZKA, M. F. Índice de qualidade da água (IQA) do reservatório Tanque Grande, Guarulhos (SP): análise sazonal e efeitos do uso e ocupação do solo. Geociências, UNESP, v. 28, n. 3, p. 305-317, 2009.

SREEDHAR, Y.; NAGARAJU, A. Groundwater quality around Tummalapalle area, Cuddapah District, Andhra Pradesh, India.
Applied Water Science, v. 7, n. 7, p. 4077-4089, 2017.

SULIS, A.; BUSCARINU, P.; SORU, O.; SECHI, J.M. Trophic State and Toxic Cyanobacteria Density in Optimization Modeling of Multi-Reservoir Water Resource Systems. Toxins, v. 6, n. 4, p. 1366-1384, 2014.

WIEGAND, M.G.; PIEDRA, J.I.G.; ARAÚJO, J.C. Vulnerabilidade à eutrofização de dois lagos tropicais de climas úmido (Cuba) e semiárido (Brasil). Eng. Sanit. Ambient, v. 21 , n. 2, p. 415-424, 2016.

Submetido em 22 de junho de 2019 Aceito em 17 de abril 2020 